\title{
Experimental study of a half-integer resonance with space-charge effects in a synchrotron
}

\author{
Tomonori Uesugi* \\ Department of Physics, University of Tokyo, Hongo, Bunkyo-ku, Tokyo 133-0033, Japan \\ Shinji Machida and Yoshiharu Mori \\ High Energy Accelerator Research Organization (KEK), Oho, Tsukuba-shi, Ibaraki-ken 305-0801, Japan
}

(Received 28 November 2000; published 8 April 2002)

\begin{abstract}
Beam losses due to half-integer resonance have been observed in the Heavy Ion Medical Accelerator in Chiba synchrotron, along with exciting the harmonic component of gradient field errors. During operation, while varying the defocusing quadrupole to cross a half-integer tune in the vertical space, the region of bare tunes which causes the half-integer resonance was evaluated. When the initial beam intensity was high, the bare tune where the beam loss occurred became higher. The beam loss occurred rapidly when the half-integer tune was crossed upward, but gradually when it was crossed downward. Those results mean that the half-integer resonance is affected by space-charge-induced tune shifts. This fact was verified experimentally for the first time. The results from a one-dimensional multiparticle simulation agreed with those characteristics. Finally, the beam-size growth and the change in distribution were studied by a simulation.
\end{abstract}

DOI: 10.1103/PhysRevSTAB.5.044201

PACS numbers: 41.75.-i, 29.27.Bd, 29.20.-c

\section{INTRODUCTION}

There are many projects involving high-intensity synchrotrons for experiments of nuclear physics, solid state physics, and biology, as well as for medical applications. In realizing such high-intensity machines, one of the most serious problems is beam loss, which causes the activation of accelerator components. However, its detailed mechanism is not clear.

One familiar model shows that the betatron tunes of individual particles are reduced by a space-charge field, and those oscillations resonate with a periodic external field $[1,2]$. However, this model is not self-consistent because it assumes that both the space-charge field and the tune of a particle are constant. In fact, it can be derived that the integer resonance does not occur even when the depressed tune of a particle is in the integer stop band associated with the dipole field errors. The reason is that the dipole field affects all of the particles equally, so that the motion of a particle moving around the center of mass does not resonate with those fields. Instead, the motion of the center of mass, namely, coherent dipole-mode oscillation, can resonate with the dipole field errors.

In 1968, Sacherer proposed a self-consistent analysis of resonances in terms of coherent motions of the particle distribution [3]. In particular, he analyzed the half-integer resonance, employing an envelope equation which describes the motion of the beam size. Based on the assumption of linear external field and a linear space-charge field, it was found that the growth of the betatron amplitude

*Present address: High Energy Accelerator Research Organization, 1-1 Oho, Tsukuba-shi, Ibaraki-ken, 305-0801, Japan. due to half-integer resonance occurs when the coherent quadrupole mode tune, not the tune of an individual particle, is near to an integer.

After Sacherer's work, the coherent motions of a beam and their tunes, not only for the second moment distribution, have drawn attention as criteria of the beam intensity limit, instead of the tunes of individual particles, namely, incoherent tunes. In 1970, Gluckstern [4] analyzed the perturbative motion of a two-dimensional cylindrical beam with uniform density by means of the Vlasov-Poisson equation and obtained the coherent modes as eigenfunctions of the space-charge potential and their tunes. Those analyses were generalized for a beam with anisotropic emittance and external focusing by Hofmann in 1998 [5].

Simulation studies have also been conducted. In 1985, Hofmann [6] simulated the integer and the fourth-order resonances when the tune approached an integer or a quarter-integer. In the simulation of integer resonance, no displacement of the beam was found when the depressed tune crossed an integer, while it occurred when the bare tune crossed an integer. Also, in a simulation of the fourthorder resonance, emittance growth did not occur, even when the depressed tune of a particle crossed the quarter integer. Thus, it was verified in the simulation that neither an integer nor a fourth-order resonance occurs at incoherent tunes. Similar simulations for half-integer and thirdorder resonances were also conducted by Machida [7], which showed that neither of them occurs when the depressed tune of a particle crosses a half-integer or a onethird integer.

Experimental studies for space-charge effect have been conducted in the Los Alamos proton storage ring [8] and in the CERN proton synchrotron (CERN PS) [9]. In Los Alamos, the beam size at a bare tune just above an integer 
was measured as a function of the beam intensity and the bare tune. The final beam size became larger when the beam intensity was high or the distance of the bare tune to the nearest integer was small. The depressed tune was calculated from the final beam size. If we take into account the Gaussian-like profile measured there, as Baartman pointed out [10], the depressed tunes of those particles with a small amplitude are below the integer value. Also, in the CERN PS, the emittance was measured near to an integer tune. The results showed that the tune of an individual particle can cross an integer value without any emittance blowup. These results from the experiments conducted in Los Alamos and in CERN mean that integer resonance does not occur at incoherent tunes. Experiments for higher order resonances do not exist thus far.

We performed an experimental study of the space-charge effects in the vicinity of a half-integer resonance and investigated the beam-size growth and detailed mechanism of the beam loss. Our experiments were to observe the beam loss when the vertical tune approaches a half-integer value.

Compared to the simplified model that Sacherer assumed, there are several issues we should take into account in order to study space-charge effects in a real synchrotron. First, in Sacherer's analysis, the external focusing structure was simplified and assumed to be constant all around a machine, which is called a smooth approximation. In addition, error fields to excite a half-integer resonance were included as a sinusoidal perturbation. In reality, almost all synchrotrons today employ an alternating gradient principle, in which focusing and defocusing are periodically located. Error fields are excited by local perturbation. Second, according to Sacherer [11], the rms envelope equation holds the same regardless of the distribution, except for a parameter (the $\lambda$ ) which very weakly depends on the distribution. Therefore, it is said that the behavior of the beam near to a half-integer resonance is well described by an analysis based on the assumption that the charge distribution is uniform. However, a slight dependence on the distribution, parameterized as $\lambda$, may cause a change in the distribution to form a positive-feedback system. Third, a real synchrotron does not have enough aperture compared to the emittance. The beam loss is likely to be determined by the available aperture. In a practical sense, if Sacherer's prediction is verified experimentally and the necessary aperture to avoid beam loss can be deduced, it should be a criterion for designing high-intensity machines. Finally, the first item of Sacherer's result implies that the condition of half-integer resonance is related to the quadrupole mode coherent tune, not the incoherent ones. We will verify this by observing the incoherent and coherent tunes.

The experiments have been carried out at the synchrotron of Heavy Ion Medical Accelerator in Chiba (HIMAC) in the National Institute of Radiological Sciences (NIRS), using a $\mathrm{He}^{2+}$ beam. In Sec. II, we present our experiments and their results. In order to understand the experimental results, we employed a one-dimensional multiparticle simulation, which is described in Sec. III. Finally, we discuss the nature of the half-integer resonance in Sec. IV, along with the results from both the experiments and the simulations.

\section{HIMAC SYNCHROTRON}

\section{A. Synchrotron}

The HIMAC accelerator [12] was constructed mainly for studying cancer therapy with heavy-ion beam, such as He, $\mathrm{C}, \mathrm{Ne}, \mathrm{Si}$, and Ar. One of the reasons that we have chosen the HIMAC synchrotron for our experiments is that the particle energy of $6 \mathrm{MeV} / \mathrm{u}$ at injection is low, so that the space-charge effect becomes strong. The other is that the large aperture of the beam pipe allows us to observe the process of beam-resonant blowup before some of particles hit the pipe wall.

Table I lists the main parameters of the HIMAC synchrotron at the injection energy. The horizontal and vertical beta functions take maximum values of 20 and $19 \mathrm{~m}$ at the center of the focusing (QF) and defocusing (QD) quadrupole magnets, respectively. In order to obtain a higher beam intensity, the HIMAC synchrotron adopts multiturn beam injection with shifting the injection bump orbit horizontally. With a typical injection current of $600 \mu \mathrm{A}$, about $10^{11} \mathrm{He}^{2+}$ particles can circulate in the synchrotron. However, when the circulating current is high, a beam loss occurs immediately after the injection. In our experiments, we decreased the beam current with meshed-plate attenuators at the injection beam line in order to observe a beam with a long lifetime.

\section{B. Beam monitors}

The circulating beam current is monitored with a dc current transformer (DCCT). The closed orbit distortion (COD) is measured with electrostatic monitors with parallel-plate electrodes near the quadrupole magnets. The horizontal and vertical beam profiles are measured at the injection beam line with multiwire beam profile monitors $(\mathrm{PRN})$. There is an emittance monitor (EMN) in the injection beam line, which is composed of a PRN monitor and a movable slit in front of it. The distribution of

TABLE I. Parameters of the HIMAC synchrotron at injection energy.

\begin{tabular}{lc}
\hline \hline \multicolumn{1}{c}{ Parameter } & Value \\
\hline Circumference & $2 \pi R=129.6 \mathrm{~m}$ \\
Lattice structure & 12 FODO, superperiod is 6 \\
Beam energy & $K_{\text {inj }}=6.0 \mathrm{MeV} / \mathrm{u}$ \\
& $(\gamma=1.06, \beta=0.113)$ \\
Revolution frequency & $261.4 \mathrm{kHz}$ \\
Repetition time & $3.3 \mathrm{sec}$ \\
\hline \hline
\end{tabular}


TABLE II. Design parameters of quadrupole monitor.

\begin{tabular}{lc}
\hline \hline \multicolumn{1}{c}{ Parameter } & Value \\
\hline Chamber material & SUS316L \\
Bore radius (electrode) & $r_{\mathrm{qm}}=104 \mathrm{~mm}$ \\
Electrode length & $\ell_{\mathrm{qm}}=400 \mathrm{~mm}$ \\
Electrode thickness & $t=3 \mathrm{~mm}$ \\
Electrode gap & $\Delta r_{\mathrm{qm}}=3 \mathrm{~mm}$ \\
Electrode capacitance & $C_{\mathrm{qm}}=192 \mathrm{pF}$ \\
Voltage gain of amplifier & $\ln G_{\mathrm{amp}}=46_{-3.0}^{+0.5 \mathrm{~dB}}$ \\
& at $1 \mathrm{kHz}-80 \mathrm{MHz}$ \\
Input impedance of amplifier & $R_{\mathrm{amp}}=1.0 \mathrm{M} \Omega$ \\
\hline \hline
\end{tabular}

particles in the horizontal or vertical phase space is obtained by measuring the profile as a function of the slit position.

In order to measure the tune shift of the coherent quadrupole mode oscillation, we installed a capacitive pickup monitor with four electrodes, namely, a quadrupole beam monitor. The parameters of the quadrupole monitor are shown in Table II. As shown in Fig. 1, the voltages at the electrodes are amplified by low-noise field-effect transistor amplifiers and processed to four output signals: horizontal $(\mathrm{H})$, vertical $(\mathrm{V})$, quadrupole $(\mathrm{Q})$, and sum $(\mathrm{S})$ channels. The output voltage of each channel is given by

$$
\begin{gathered}
V_{\mathrm{H}}=-4 \sqrt{2} \lambda A\langle\xi\rangle, \\
V_{\mathrm{V}}=-4 \sqrt{2} \lambda A\langle\eta\rangle, \\
V_{\mathrm{Q}}=-8 \lambda A\left(\left\langle\xi^{2}\right\rangle-\left\langle\eta^{2}\right\rangle\right), \\
V_{\mathrm{S}}=-2 \pi \lambda A,
\end{gathered}
$$

where $\lambda$ is the line density and $\xi-\eta$ is the horizontal and vertical position normalized in the aperture radius. In this paper, the brackets, in general, denote the averaged value. The factor $A$ is calculated by

$$
A=-G \frac{\ell_{\mathrm{qm}}}{2 \pi C_{\mathrm{qm}}},
$$

where $G=10^{4.6}$ is the gain of the amplifier and $\ell_{\mathrm{qm}}$ and $C_{\mathrm{qm}}=192 \mathrm{pF}$ are the length and the capacitance of an electrode. The $\mathrm{H}$ and $\mathrm{V}$ channels represent the horizontal and vertical positions of the beam center. Coherent dipolemode oscillations are seen in these channel outputs. In the Q-channel output, the first order dipole mode $(\langle\xi\rangle)$ signals ideally vanish and the coherent quadrupole mode signals are emphasized. This type of beam monitor has also been used in LEAR at CERN to measure the quadrupole mode tune [13].

\section{Stop band control}

The stop band width of the half-integer resonance was controlled by a pair of additional quadrupole magnets located at exactly opposite sides of the synchrotron. It has been named the defocusing quadrupole shifter (QDS) [14]. We excited the two magnets of the QDS in counterphase so that the stop band width could be controlled without changing the tunes.

The stop band half-width of the half-integer resonance is calculated by

$$
\frac{\delta \nu_{y}}{2}=\sqrt{\left(a I_{\mathrm{QDS}}+\kappa_{1}\right)^{2}+\kappa_{2}^{2}},
$$

with

$$
\begin{gathered}
\kappa_{1}=\frac{1}{4 \pi} \oint \beta_{y}(s) K(s) \cos \left[2 i \phi_{y}(s)\right] d s, \\
\kappa_{2}=\frac{1}{4 \pi} \oint \beta_{y}(s) K(s) \sin \left[2 i \phi_{y}(s)\right] d s, \\
a=\frac{1}{4 \pi} \frac{2 \beta_{y} K_{\mathrm{QDS}} \Delta \ell}{I_{\mathrm{QDS}}} .
\end{gathered}
$$

Here, $s$ is the longitudinal coordinate, $\phi_{y}(s)$ is the vertical betatron phase advance measured from the position of one of the QDS magnets,

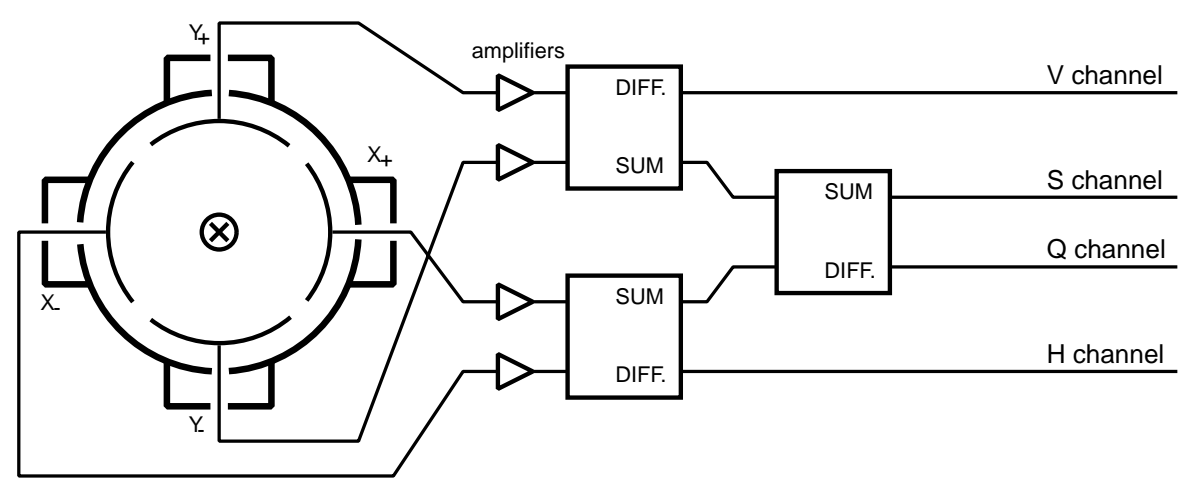

FIG. 1. Data processing system of the quadrupole beam monitor. The voltages of four electrodes $\left(\mathrm{X}^{+}, \mathrm{X}^{-}, \mathrm{Y}^{+}, \mathrm{Y}^{-}\right)$are amplified and processed into four channels $(\mathrm{V}, \mathrm{H}, \mathrm{Q}, \mathrm{S})$. The hybrid junctions output the sum (SUM) and difference (DIFF) of the input signals. 


$$
K(s)=\frac{1}{B_{0} \rho}\left[\frac{\partial B_{y}(s)}{\partial x}\right]_{0}=\frac{1}{B_{0} \rho}\left[\frac{\partial B_{x}(s)}{\partial y}\right]_{0},
$$

is the normalized field-gradient error at the center of the aperture, and $B_{0} \rho$ is the rigidity of a beam in the bending magnets. The space-charge effects are not included here. We assumed that the thickness of the QDS magnet is much less than the wavelength of the beta function, so that the vertical beta function is constant there. In our case, the parameter $a$ is estimated to be 0.0014 [1/A], while $\kappa_{1,2}$ should be determined by measurements.

$$
\left[\begin{array}{l}
d \nu_{0 x} \\
d \nu_{0 y}
\end{array}\right]=\left[\begin{array}{c}
+0.060 \pm 0.001 \\
-0.0098 \pm 0.0005
\end{array}\right.
$$

where the subscript " 0 " denotes the value at the zerocurrent limit. The coefficient $d \nu_{0 y} / d I_{\mathrm{QD}}$ was used to evaluate $\nu_{0 y}$ at an arbitrary time when $I_{\mathrm{QD}}$ was varied. In the presence of a strong space-charge field or external error field, the betatron tunes are shifted and the $\left(\nu_{0 x}, \nu_{0 y}\right)$ are no longer the actual tunes. We call $\left(\nu_{0 x}, \nu_{0 y}\right)$ the unperturbed bare tunes.

Next, we measured the space-charge-induced tune shifts of the coherent quadrupole and dipole-mode oscillations. Here, we employed a radio-frequency quadrupole (RFQ) [15] to emphasize the quadrupole mode sidebands. The RFQ was composed of four magnetic poles and was excited with broadband signal filling of $\sim 20 \mathrm{MHz}$. The input power was kept at less than $100 \mathrm{~W}$ so as to minimize the effects on the betatron frequencies. The unperturbed bare tune was $(3.68,3.13)$.

In the Q-channel output of the quadrupole monitor, two types of sidebands were observed at the twice vertical betatron frequency measured from every harmonic of revo-

(A)

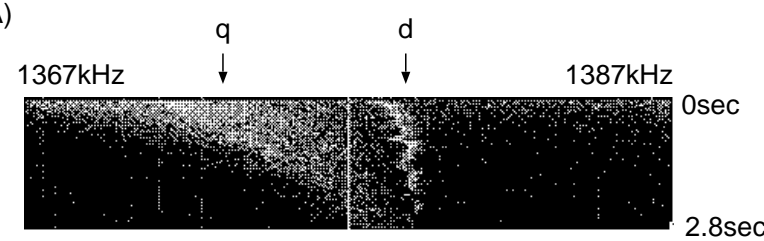

(B)

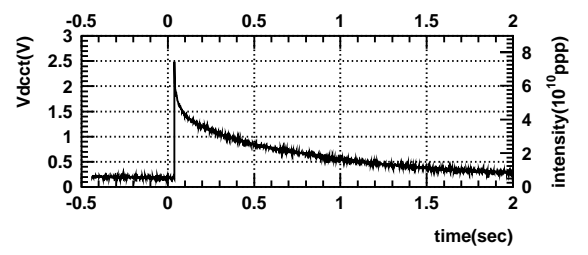

FIG. 2. Space-charge tune shift of a quadrupole-mode betatron oscillation in a $\mathrm{He}^{2+}$ coasting beam at the injection energy. (A) The spectrogram (two-dimensional plot of the spectrum versus time) of the Q-channel output from injection (top) to $2800 \mathrm{msec}$ (bottom) after it. The frequency range is $1377 \pm$ $10 \mathrm{kHz}$. A second harmonic of the dipole-mode sideband " $2 d$ " and a quadrupole mode sideband $q$ can be seen. (B) Intensity of the same beam.

\section{Tunes and tune shifts}

We have measured the frequencies of the betatron sidebands to find the relation between the tunes and the strengths of the quadrupole magnets. The beam intensity was limited to be less than $0.6 \times 10^{10} \mathrm{ppp}$ with a meshedplate attenuator placed at the injection beam line, in order to exclude any space-charge effects on the betatron tunes. The measured tunes showed a linear dependence on the quadrupole-magnet currents. The fitting results of the linear coefficients are

$\left.\begin{array}{l}-0.00766 \pm 0.0001 \\ +0.05966 \pm 0.00001\end{array}\right]\left[\begin{array}{l}d I_{\mathrm{QF}} \\ d I_{\mathrm{QD}}\end{array}\right]$,

lution frequency. An example is shown in Fig. 2(A). The center frequency of Fig. 2(A), $1377 \mathrm{kHz}$, is $-1+2 \times$ 3.13 times the revolution frequency. At that time, beam intensity was decreased as shown in Fig. 2(B), because of the RFQ perturbation. A signal " $q$ " near the second harmonic of a vertical dipole mode betatron sideband " $2 d$ " was observed. The frequency of the $q$ signal became closer to that of $2 d$ as the beam intensity decreased, and the two frequencies agreed at the low-current limit. Thus, the signal $q$ was identified as a coherent quadrupole-mode sideband. The intensity dependence of the quadrupole mode tune was

$$
\frac{\Delta \nu_{q}}{2}=(0.0028 \pm 0.00004) / 10^{10}[\mathrm{ppp}] \times N \text { measured }
$$

where $N$ is the number of particles in the synchrotron. The quadrupole-mode tune shift is defined by

$$
\frac{\Delta \nu_{q}}{2}=\frac{f_{0,2 y}-f_{q}}{2 f_{0}}
$$

where $f_{0}$ is the revolution frequency, $f_{q}$ is the frequency of the quadrupole mode sideband, and $f_{0,2 y}$ is the frequency

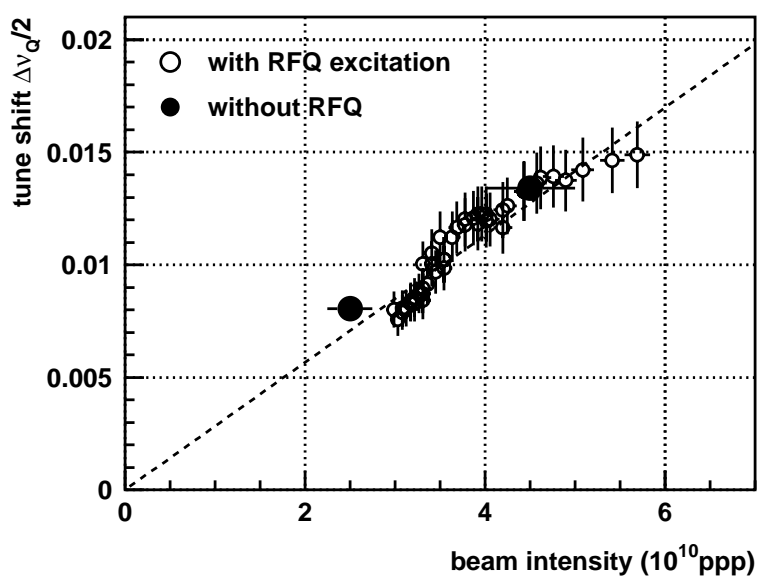

FIG. 3. Space-charge-induced tune shift of vertical quadrupole mode coherent oscillation. 
of the second-harmonic dipole-mode sideband in the limit of low current. The two data points in Fig. 3 show the tune shifts for different beam intensities in the absence of RFQ excitation, which agree with Eq. (12). A similar measurement for the coherent dipole tune shift in vertical space resulted in

$\Delta \nu_{d}=(0.0009 \pm 0.00001) / 10^{10}[\mathrm{ppp}] \times N$ measured

which roughly agrees with the value expected with the half-integer formula of a Laslett tune shift [16].

\section{E. Emittance}

We have observed the phase-space distributions of an injector beam with an EMN monitor. The 95\% emittances, defined by the phase-space area where the density is higher than $5 \%$ of the maximum, are about $(10.3,8.8) \pi \mathrm{mm} \mathrm{mrad}$. The beam profiles were also measured by a PRN monitor at the end of the injection beam line, where the beta function is $1.25 \mathrm{~m}$ in both the horizontal and the vertical direction. The rms sizes of those profiles were $1.79 \mathrm{~mm}$ in the horizontal and $1.45 \mathrm{~mm}$ in the vertical direction, which correspond to an rms emittance of $(2.6,1.7) \pi \mathrm{mm}$ mrad.

The tune shift of the quadrupole mode mainly comes from the defocusing field due to space charge. It thus reflects the charge density of the beam. We calculated the vertical quadrupole mode tune shift as a function of the vertical emittance and compared it with the experimental value in order to estimate the vertical rms emittance in the synchrotron.

In a beam with an elliptic cross section, the vertical quadrupole mode tune shift is written approximately by [17]

$$
\frac{\Delta \nu_{q}}{2}=\frac{1}{4}\left(3-\frac{\tilde{y}}{\tilde{x}+\tilde{y}}\right) \Delta \nu_{y}
$$

with

$$
\Delta \nu_{y}=\frac{1}{4 \pi \nu_{0 y}} \frac{N r_{0} R}{\beta^{2} \gamma^{3}} \frac{1}{\tilde{y}(\tilde{x}+\tilde{y})},
$$

where the tilde denotes the rms value, $N$ is the number of particles in the whole ring, $r_{0}$ is the classical radius of the particle, and $R$ is the average radius of the synchrotron. By replacing

$$
\tilde{x}=\sqrt{E_{x} \beta_{x}}, \quad \tilde{y}=\sqrt{E_{y} \beta_{y}}
$$

in Eqs. (15) and Eq. (16), the quadrupole-mode tune shift can be written by rms emittances $E_{x}-E_{y}$ and beta functions $\beta_{x}-\beta_{y}$. We calculated the quadrupole-mode tune shift as a function of $E_{y}$. Here, the horizontal rms emittance was taken to be $E_{x}=(120 \pm 14) \pi \mathrm{mm}$ mrad, based on the assumptions that the full emittance equals an acceptance of $480 \pi \mathrm{mm}$ mrad and the density is uniform because of multiturn injection. The systematic error in $E_{x}$ comes from the dispersion effect of $2.9 \mathrm{~mm}$ at maximum. Comparing the result with the measured value of Eq. (12), we obtained a vertical rms emittance of $(2.0 \pm 0.2) \pi \mathrm{mm} \mathrm{mrad}$, which is slightly larger than the measured value of $1.7 \pi \mathrm{mm} \mathrm{mrad}$ at the injection beam line.

\section{F. Resonance experiments}

We have observed the beam loss in the HIMAC synchrotron while varying the QD current linearly to bring about a vertical tune across a half-integer value. The QDS was also excited so as to control the stop band width of the half-integer resonance. For a comparison, similar data with low-intensity beams were taken. The beam current was recorded every $10 \mathrm{msec}$ with a digital oscilloscope synchronized with HIMAC operation.

Table III lists the parameters. We took two patterns of QD excitation for upward and downward half-integer crossing. The QF current was fixed at 77.5 A, which optimizes the injected current at around $\nu_{y}=3.5$. The initial QD current was chosen to be sufficiently far from the half-integer tune to maintain high intensity injection for all ranges of QDS current. The initial vertical bare tune was measured with a very low current. The ramping rate of the QD current was set to be very slow, as long as the resonance was crossed in $2500 \mathrm{msec}$, taking a $800 \mathrm{msec}$ recovery time of the QD current into account. The excitation patterns of the QD magnets are shown in Fig. 4. The bare tune at time $t$ can be obtained by

$$
\begin{aligned}
\nu_{0 y}(t)=\nu_{0 y}(0)+0.05966[1 / \mathrm{A}] \times \frac{d I_{\mathrm{QD}}}{d t} t, \\
0<t<2500[\mathrm{msec}] .
\end{aligned}
$$

Figure 5 shows the typical beam-loss waveforms. The qualitative behaviors of the beam loss were obviously different between the downward and upward half-integer crossings. In the case that the resonance was crossed downward, the beam loss occurred gradually, while it

TABLE III. The conditions of the resonance cross experiments and multiparticle simulation.

\begin{tabular}{ccc}
\hline \hline \multicolumn{2}{c}{$(\mathrm{A})$ downward } \\
\hline Parameter & Simulation & Experiment $\left(I_{\mathrm{QDS}}=-2 \mathrm{~A}\right)$ \\
\hline$\nu_{0}$ (initial) & 3.5157 & 3.567 \\
$d \nu_{0} / d N_{t}$ & $-1.126 \times 10^{-6}$ & $-1.126 \times 10^{-7}$ \\
$\delta \nu$ & 0.01 & 0.01 \\
$\Delta \nu_{q} / 2$ & 0.0059 & 0.0052 \\
\hline
\end{tabular}

(B) upward

\begin{tabular}{ccc}
\hline Parameter & Simulation & Experiment $\left(I_{\mathrm{QDS}}=-2 \mathrm{~A}\right)$ \\
\hline$\nu_{0}$ (initial) & 3.4951 & 3.458 \\
$d \nu_{0} / d N_{t}$ & $+1.126 \times 10^{-7}$ & $+1.126 \times 10^{-7}$ \\
$\delta \nu$ & 0.01 & 0.01 \\
$\Delta \nu_{q} / 2$ & 0.0066 & 0.0064 \\
\hline \hline
\end{tabular}


(A)downward crossing

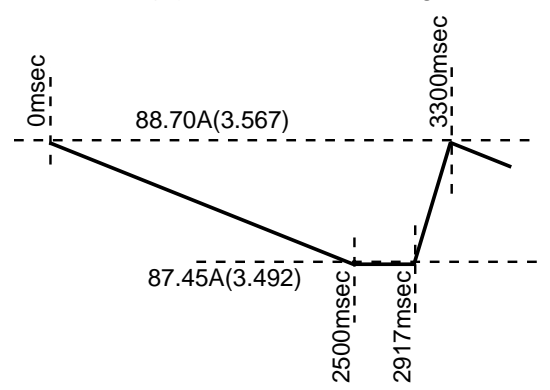

(B)upward crossing

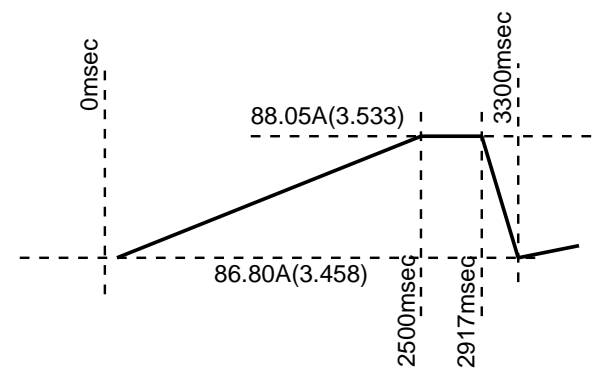

FIG. 4. Excitation patterns of QD magnets in (A) downward and (B) upward resonance crossing experiments. The horizontal axis shows the time after the master trigger and the vertical axis shows the QD current.

occurred rapidly in the other case. Those characteristics were independent of the current of QDS. The gradual loss behavior in the downward cases can be explained by a detuning effect due to decreasing beam intensity. If a part of the beam is lost, the space-charge effect is decreased and the depressed tunes of particles become higher. This effect makes the tune escape from the half-integer value when the depressed tune is above it, so that the beam loss occurs gradually. On the other hand, in the case of upward crossing, the detuning effect due to beam loss makes the depressed tune approach the half-integer, and the beam loss occurs rapidly.

Figure 6 shows the bare tune at the time $t_{c}$ and $t_{f}$ when the beam loss began or was finished. Those times are
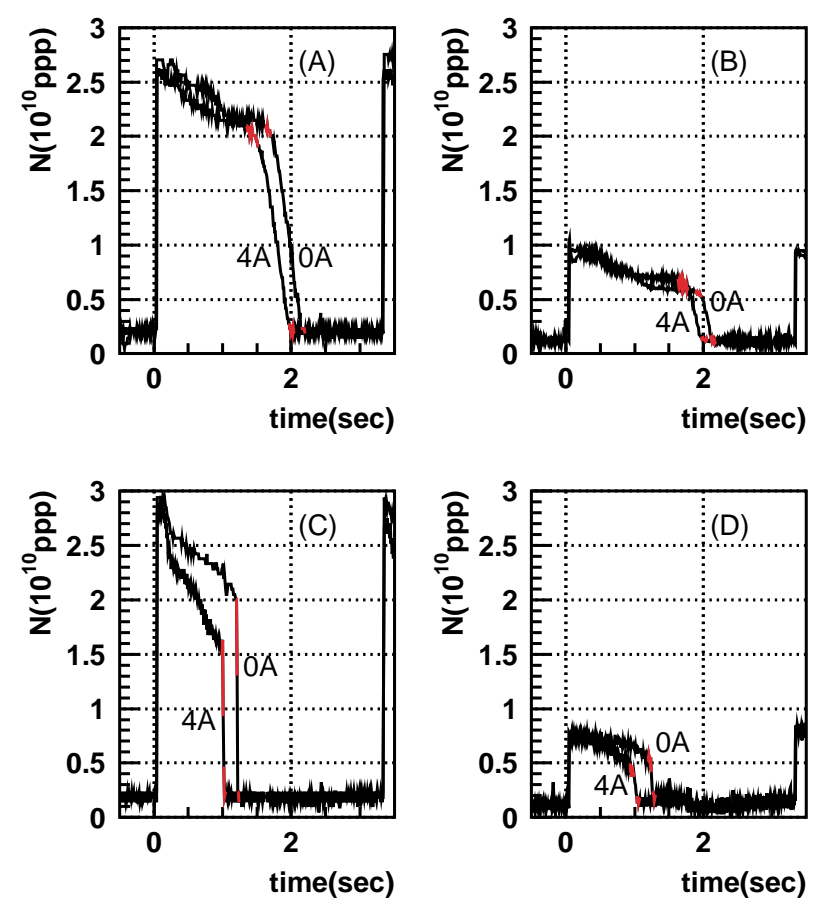

FIG. 5. (Color) Typical beam loss waveforms when the resonance line was crossed (A),(B) downward or (C),(D) upward. The initial beam intensity was decreased with an attenuator in cases (B) and (D). The data for $I_{\mathrm{QDS}}=0 \mathrm{~A}$ and $4 \mathrm{~A}$ are shown. The red parts of each waveform define the tunes where beam loss occurred and was finished. defined as the red regions of the waveforms in Fig. 5, where the slope of the beam current is quickly changing. The bare tune at a given time is calculated by Eq. (18). The major sources of systematic error on $\nu_{0 y}\left(t_{c, f}\right)$ are the ambiguity of reading the beam loss time, $t_{c, f}$, and the accuracy of the initial tune measurement.

If we neglect the space-charge effect in lower current data, which is of the order of 0.001 in tune shift of a particle, the $\nu_{0 y}\left(t_{c}\right)$ of those data should correspond to the edge of the stop band at each strength of the gradient error. The top points of the wedge-shaped function of $\nu_{0 y}\left(t_{c}\right)$ indicate the natural component of the gradient field error. The fitting parameters in Eq. (6) are

$$
\left(\begin{array}{c}
\kappa_{1} \\
\kappa_{2} \\
a
\end{array}\right)=\left(\begin{array}{c}
(7.1 \pm 0.5) \times 10^{-3} \\
(3.1 \pm 0.8) \times 10^{-3} \\
(1.7 \pm 0.1) \times 10^{-3}
\end{array}\right)
$$

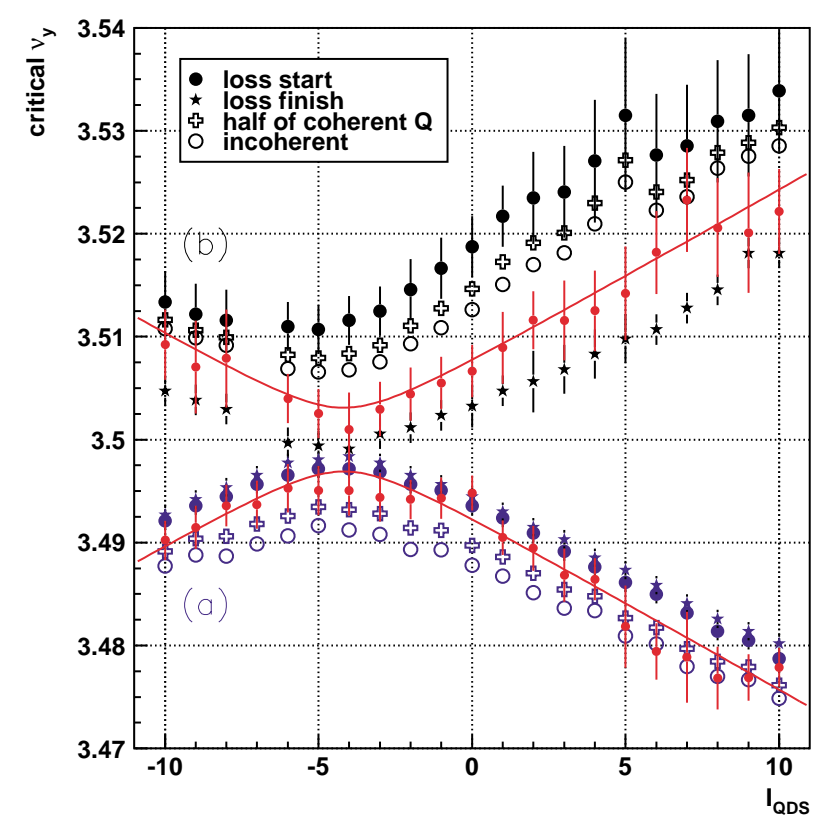

FIG. 6. (Color) Bare tunes corresponding to the times where beam loss began $\left(t_{c}\right.$, solid circles) and was finished $\left(t_{f}\right.$, stars). The open circles and cross symbols show the incoherent (with approximation of linear space-charge field) and half of coherent tunes corresponding to $t_{c}$. The red symbols show low-intensity data. 
where $a$ is in units of [1/A]. The slope $a$ roughly agrees with the expected value of 0.0014 [1/A]. It proves that the beam losses in our experiments are surely caused by half-integer resonance due to a QDS field.

In the cases of high beam intensity, the tune where beam loss occurred was shifted upward. That intensity dependence, as well as the beam-loss behavior, is direct evidence of the space-charge effect changing the condition of the resonant beam loss.

\section{G. Summary}

We have observed the beam loss due to a half-integer resonance, with varying the defocusing quadrupole to cross a half-integer betatron tune in vertical space. The tune where beam loss occurred depended on the strength of an additional gradient field, and that dependence agreed with the expected value, if we assumed a natural component of the gradient field error.

When the injected current was high, the tune where beam loss occurred became higher in terms of an unperturbed bare tune. That was the space charge effect on the condition of beam loss due to a half-integer resonance; we verified it experimentally for the first time.

The behaviors of beam losses were different between upward and downward half-integer crossings. When the half-integer tune was crossed downward, the beam loss occurred gradually, while it occurred rapidly in upward crossing. Those behaviors can be understood in terms that the beam loss weakens the space-charge effect to shift the depressed tune upward.

\section{MULTIPARTICLE SIMULATIONS}

\section{A. Simulation method}

We employed a one-dimensional multiparticle simulation with space-charge effects in order to understand the results of the resonance-crossing experiments. Because of the horizontal multiturn injection and anisotropic aperture of a vacuum chamber at quadrupole magnets, the HIMAC beam has about 20 times larger emittance in horizontal space compared with the vertical space. In such a beam, the vertical self-field is almost independent of the horizontal position. In addition, coupling between the horizontal and vertical motions must be small because of the asymmetric tunes. Therefore, we separated the vertical motion from the horizontal motion, as if the beam has an infinite horizontal beam size with a uniform density. That is called the sheet beam approximation.

As a model of the HIMAC synchrotron, we used a lattice with 12 FODO cells of equally spaced magnets and two additional magnets as QDS. The QDS magnets are located at the middle points of the QD and QF magnets. Because the length of the magnets was negligible, we took the thinlens approximation.
In order to simplify the equation of motion, we defined the normalized coordinates of longitudinal $(s)$ and transverse $(y)$ directions by

$$
\begin{gathered}
\hat{s}=\frac{48}{2 \pi R} s, \\
\dot{y}=\sqrt{\frac{48}{2 \pi R E_{y}}} y,
\end{gathered}
$$

respectively, where $2 \pi R=129.6 \mathrm{~m}$ is the circumference of the HIMAC synchrotron, 48 is 2 times the number of focusing quadrupole magnets, and $E_{y}=2.0 \pi \mathrm{mm} \mathrm{mrad}$ is the vertical rms emittance of a HIMAC beam in the absence of a perturbation from the QDS magnets. In this scale, the circumference becomes 48 and the initial emittance becomes unity. Hereafter, we use those coordinates, written as $s-y$ for $s-y ́$.

The equation of motion in the drift space between magnets is

$$
y^{\prime \prime}= \pm \sqrt{3} K_{\mathrm{sc}} n(y),
$$

where $K_{\mathrm{sc}}=2 \pi N_{1} r_{0} / \sqrt{3} \beta^{2} \gamma^{3}$ is the parameter of spacecharge forces, $n(y)$ is the fraction of particles inside $\pm y$, and $N_{1}$ is the density in the horizontal plane. The dual sign in Eq. (22) is positive when $y>0$ and negative otherwise. At the location of quadrupole magnets, an impulse of $Q^{2}$ was interpolated on $y^{\prime}$ twice (to monitor the coordinates of particles at the center of magnets) without changing $y$. The parameter $2 Q^{2}$ represents the field gradient of the main $\left(2 Q_{q}^{2}\right)$ or error $\left(2 Q_{e}^{2}\right)$ magnets, and is given by

$$
2 Q^{2}=\frac{2 \pi R}{48} \frac{1}{B_{0} \rho}\left(\frac{\partial B_{x}}{\partial y}\right) \Delta \ell,
$$

where $\Delta \ell$ is the length of those magnets and $B_{0} \rho$ is the rigidity of a beam. In dynamic resonance crossing operations, the strength of the main magnets $\left(Q_{q}\right)$ was varied in a linear function of the revolution number. Once a particle exceeds the aperture limit $\left(y_{\text {lim }}\right)$, it should be removed.

The initial state of macroparticles was specified by the number of macroparticles $\left(N_{p}\right)$, Twiss parameters $(\alpha, \beta)$ at $s=0$, mismatch parameter $\left(X_{\mathrm{mis}}\right)$, and a distribution type - uniform (UN) or parabolic (PA) distribution. In the case of $\alpha(0)=0, \beta(0)=1$, and $X_{\text {mis }}=1$ (no mismatch), our distribution function can be written as shown in Table IV. A general expression can be obtained from those normalized distributions by the transformation

$$
\left(\begin{array}{c}
y \\
y^{\prime}
\end{array}\right)=\left(\begin{array}{cc}
\cos \phi & -\sin \phi \\
\sin \phi & \cos \phi
\end{array}\right)\left(\begin{array}{cc}
X_{\mathrm{mis}} / F_{1} & 0 \\
0 & 1 / F_{2}
\end{array}\right)\left(\begin{array}{c}
y \\
y^{\prime}
\end{array}\right)_{\text {norm }},
$$

where

$$
\begin{aligned}
& F_{1}=\sqrt{\gamma \cos ^{2} \phi+2 \alpha \sin \phi \cos \phi+\beta \sin ^{2} \phi}, \\
& F_{2}=\sqrt{\gamma \sin ^{2} \phi-2 \alpha \sin \phi \cos \phi+\beta \cos ^{2} \phi},
\end{aligned}
$$


TABLE IV. Normalized expressions of distribution functions in our simulation. The symbols UN and PA stand for uniform and parabolic distributions, respectively. $f(r)$ is the density in phase space as a function of radius $r^{2}=y^{2}+y^{\prime 2}$, and $p(y)$ is the projection on real space.

\begin{tabular}{cccc}
\hline \hline Symbol & $f(r)$ & $p(y)$ & $y_{\max }$ \\
\hline $\mathrm{UN}$ & $\frac{1}{6 \pi} \frac{1}{\sqrt{1-r^{2} / 3}}$ & $\frac{1}{2 \sqrt{3}}$ & $\sqrt{3}$ \\
$\mathrm{PA}$ & $\frac{1}{3 \pi}\left(1-r^{2} / 6\right)$ & $\frac{8}{3 \sqrt{6} \pi}\left(1-y^{2} / 6\right)^{3 / 2}$ & $\sqrt{6}$ \\
\hline \hline
\end{tabular}

$$
\phi=\frac{1}{2} \tan ^{-1} \frac{2 \alpha}{\gamma-\beta},
$$

and

$$
\gamma=\frac{1+\alpha^{2}}{\beta} .
$$

The Twiss parameters are evaluated by solving the envelope equation, which is described in the next subsection.

In the simulation, once per turn we recorded the second moments of the particle distribution and the coordinates of some particles that we chose, at the point corresponding to $s=0$. The coordinates of all particles were recorded every 2500 turns. The incoherent tune of a particle at the $N_{t}$ th turn was obtained by picking up the peak frequency of the spectrum,

$$
A_{j}(\nu)=\left|\sum_{k=N_{t}-d N_{t} / 2}^{N_{t}+d N_{t} / 2} y_{j, k} \exp (2 \pi i \nu k)\right|^{2},
$$

where $y_{j, k}$ is the coordinate of the $j$ th particle at the $k$ th turn and $d N_{t}$ is the turn number used to evaluate the tune. The assignment of tunes for a peak frequency has the ambiguity of the integer part. For example, a particle with tune $\nu=3.6$ has peaks on its spectrum at $\nu=N \pm 0.4$. Since the operating points were very close to 3.5 in our case, the integer part of 3 was chosen. Whether the tune is higher or lower than 3.5 was judged by the phase relations between $y_{j, k}$ and $y_{j, k}^{\prime}$, checking the signs of

$$
D_{\text {inc }}=y_{j, k} y_{j, k+1}^{\prime}-y_{j, k+1} y_{j, k}^{\prime} \text {. }
$$

When $D_{\text {inc }}>0\left(D_{\text {inc }}<0\right)$ for the majority of $k$, we took $4.0>\nu_{j}>3.5\left(3.5>\nu_{j}>3.0\right)$. On the other hand, the quadrupole mode coherent tune is obtained by the track of $\tilde{y}$. The coherent spectrum is

$$
A(\nu)=\left|\sum_{k=N_{t}-d N_{t} / 2}^{N_{t}+d N_{t} / 2} \tilde{y}_{k} \exp (2 \pi i 2 \nu k)\right|^{2} .
$$

In this case, the phase relation between $\tilde{y}$ and $\left\langle y y^{\prime}\right\rangle$ is used to choose the region of a coherent tune. The definition of the determinant is

$$
\begin{aligned}
D_{\mathrm{coh}}= & {\left[\left(\tilde{y}_{k+2}-\tilde{y}_{k+1}\right)-\left(\tilde{y}_{k+1}-\tilde{y}_{k}\right)\right] } \\
& \times\left(\left\langle y y^{\prime}\right\rangle_{k+1}-\left\langle y y^{\prime}\right\rangle_{k}\right) .
\end{aligned}
$$

Using the histogram of the real-space distribution, we defined the $99 \%, 90 \%$, and $75 \%$ beam sizes, inside which those amounts of particles exist. The stepwise distribution function of the histogram data was used directly without smoothing it. We also recorded those beam sizes every turn.

\section{B. Numerical solution of the envelope equation}

The envelope equation corresponding to Eq. (22) is

$$
\tilde{y}^{\prime \prime}-\frac{1}{\tilde{y}^{3}}=\frac{\left\langle\sqrt{3} y K_{\mathrm{sc}} n(y)\right\rangle}{\tilde{y}} .
$$

According to Sacherer [11] the right-hand side of Eq. (33) nearly equals $K_{\mathrm{sc}}$ independent of the distribution; we thus used this replacement. We integrated the above equation to find a matched solution, using the fourth-order Runge-Kutta method [18] for $\tilde{y}$ and $\tilde{y}^{\prime}$. An impulse of $\pm 2 Q^{2} \tilde{y}$ was applied on $\tilde{y}^{\prime}$ at the magnets. We required a matched solution in which the change in both $\tilde{y}$ and $\tilde{y}^{\prime}$ in one revolution was within $0.001 \%$. Because the matched solution, written by $\tilde{y}_{m}(s)-\tilde{y}_{m}^{\prime}(s)$, is related to the Twiss parameter,

$$
\begin{gathered}
\alpha(s)=-\tilde{y}_{m}(s) \tilde{y}_{m}^{\prime}(s), \\
\beta(s)=\tilde{y}_{m}^{2} .
\end{gathered}
$$

The betatron tune can be calculated by

$$
\nu=\frac{1}{2 \pi} \oint \frac{d s}{\tilde{y}_{m}(s)^{2}},
$$

where the nonlinearity of the space-charge field is neglected. In a beam with a general distribution, the tunes of individual particles are spread around the above value.

\section{Simulation parameters}

Table III lists the simulation parameters together with the experimental parameters for $I_{\mathrm{QDS}}=-2 \mathrm{~A}$. In order to save calculation time, the initial tunes were chosen to be nearer to the half-integer than in the actual experiments. In addition, the ramping rate of the defocusing magnets was 10 times larger than in a simulation of the downward resonance approach. Similar simulations with more than 10 times larger ramping rate were also performed to verify that the rms beam size depends only on the tune, regardless of its ramping rate.

At first, the unperturbed bare tune $\left(\nu_{0}\right)$ is related to $Q_{q}$ as

$$
\begin{aligned}
& 2 \cos \frac{2 \pi \nu_{0}}{12}=\operatorname{tr}[\left.M_{O} M_{D} M_{O} M_{F}\right] \\
&=\operatorname{tr}\left[\left(\begin{array}{ll}
1 & 2 \\
0 & 1
\end{array}\right)\left(\begin{array}{cc}
1 & 0 \\
2 Q_{q}^{2} & 1
\end{array}\right)\left(\begin{array}{ll}
1 & 2 \\
0 & 1
\end{array}\right)\right. \\
&\left.\times\left(\begin{array}{cc}
1 & 0 \\
-2 Q_{q}^{2} & 1
\end{array}\right)\right],
\end{aligned}
$$

where $M_{F}$ and $M_{D}$ are the transfer matrices of the focusing and defocusing magnet and $M_{O}$ is that of the drift space 
between them. We calculated Eq. (37) to find that it can be approximated by a linear equation,

$$
\nu_{0}=15.8032 Q_{q}-6.4530,
$$

in the region $3.45<\nu_{0}<3.55$ of interest. We used Eq. (38) to evaluate the initial values of $Q_{q}$ and $d Q_{q} / d N_{t}$.

If a field gradient error $\left(Q_{e}\right)$ exists, the tune is shifted from the unperturbed one $\left(\nu_{0}\right)$, or the oscillation diverges when $\nu_{0}$ is in the half-integer stop band. The relation between the stop band half-width $(\delta \nu / 2)$ and $Q_{e}$ is

$$
\frac{\delta \nu}{2}=\frac{1}{4 \pi} \beta_{e} 2 Q_{e}^{2} 2,
$$

where $\beta_{e}$ is the unperturbed betatron amplitude evaluated at the error magnets. The value of $\beta_{e}$ was obtained by the matched solution of the envelope equation to be $2.84 \pm$ 0.005 for $3.49<\nu_{0}<3.52$. Equation (39) was used to determine the value of $Q_{e}$ corresponding to the given stop band width.

We evaluated the value of $K_{\mathrm{sc}}$ to induce the same quadrupole mode tune shift as in the experiments. Figure 7 shows the depressed tunes for $K_{\mathrm{sc}}=0.0014$ and 0.0005 , calculated by Eq. (36) with the matched rms envelope. The tune shifts were 6.6 times $K_{\mathrm{sc}}$ in both cases. In a sheet beam, the coherent quadrupole mode tune shift is $3 / 4$ times the incoherent one, so that

$$
\frac{\Delta \nu_{q}}{2}=\frac{3}{4} \times 6.6 K_{\mathrm{sc}} .
$$

We took $K_{\text {sc }}=0.001187$ for the downward and 0.001319 for the upward crossing simulation, which corresponded to $\Delta \nu_{\mathrm{q}} / 2=0.0059$ and 0.0066 , respectively.

According to an estimation from the quadrupole mode tune shift, the vertical rms emittance in the absence of perturbation is $2.0 \pi \mathrm{mm}$ mrad. Because we took a vertical aperture limit of $22 \mathrm{~mm}$, while taking account of a $10 \mathrm{~mm}$ COD, the parameter $y_{\text {lim }}$ was 10 .

The integrated distribution function $(n(y))$ was calculated by dividing the vertical space ( $y$ ) in $N_{d}$ and counting the number of macroparticles within every division.

We divided the vertical space $(y)$ into 30 parts to calculate the integrated distribution function $(n(y))$. The value
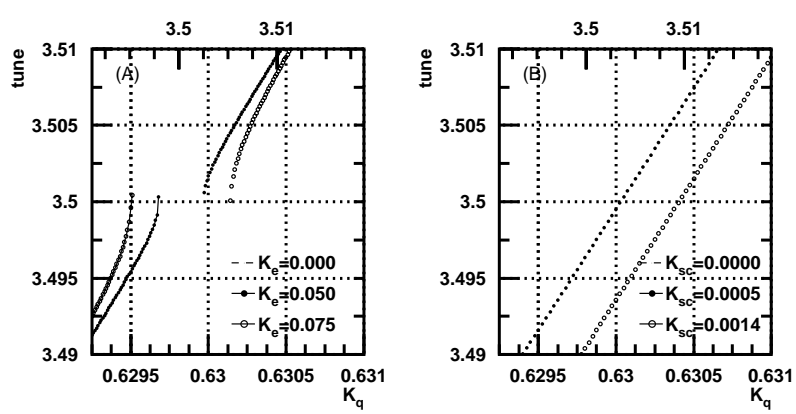

FIG. 7. Betatron tune in the presence of (A) gradient error field or (B) space-charge field. of $n(y)$ between grid points was smoothly interpolated with a quadratic function. Since the minimum value of the beta function is 1 , a transverse size of a beam with parabolic distribution is larger than 3.6 times the division size.

\section{Simulation results}

First, we show the results of a simulation without any space-charge effects $\left(K_{\mathrm{sc}}=0\right)$. In this case, the equation of motion for all particles is linear and all particles have the same tune regardless of the amplitude, beam size, or distribution.

Figure 8(B) shows the rms beam size at $s=0$ every turn. The resonant growth can be seen in both sides of the half-integer tune. The matched solution of envelope oscillation, shown by the dashed line, agrees with the simulation result as a function of $Q_{q}$. This means that the operation with changing the tune has been done adiabatically.

The quadrupole mode coherent tune and the tunes of five particles with different amplitudes (emittances) were calculated with a 1000-turn Fourier transformation.
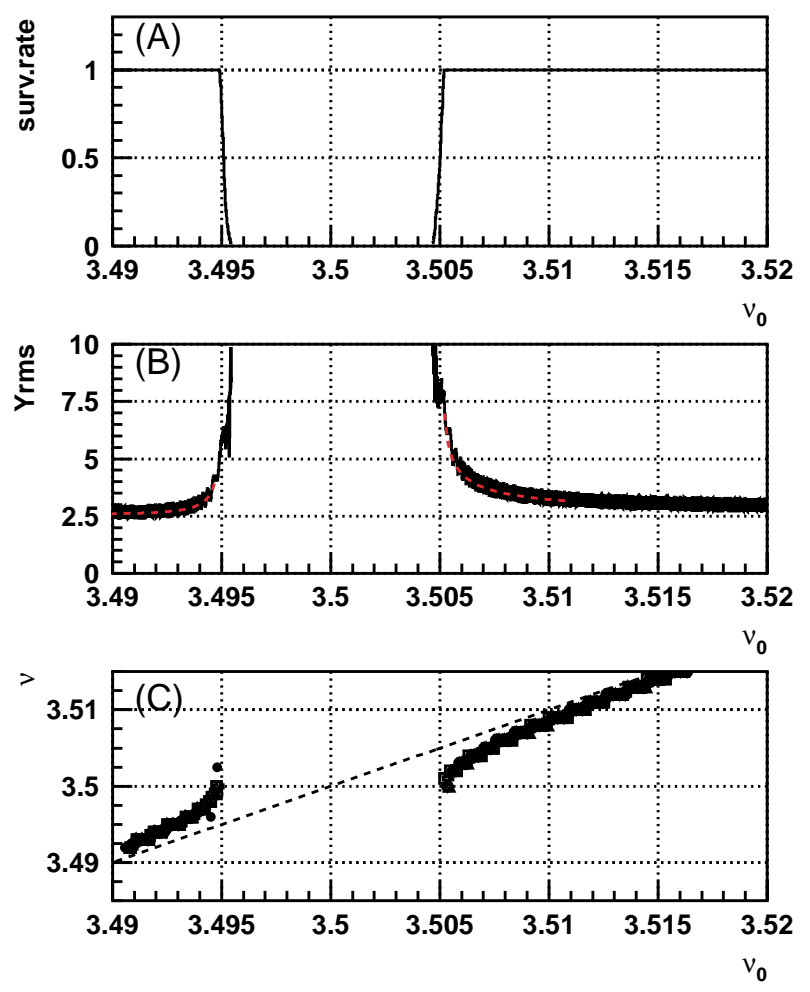

FIG. 8. (Color) Simulation results without the space-charge effect. The betatron tunes are varied to cross the half-integer resonance upward or downward. The horizontal axis corresponds to the bare tune by Eq. (38). (A) Ratio of survival macroparticles. (B) rms size of simulated beam (solid line) and those obtained by the envelope equation (dashed line). (C) Observed tunes: the solid circles show the half of quadrupole mode coherent tune and the white characters show the tunes of single particles. The dashed line is an unperturbed tune, which is identical to $\nu_{0}$. 
Figure $8(\mathrm{C})$ shows that they all agree with the tune calculated from the matched solution of the envelope equation by Eq. (36). The stop band width is 0.01 , as expected.

Figures 9 and 10 show the simulation results with the space-charge effect for a uniform and a parabolic beam, respectively. As shown in Figs. 9(A) and 10(A), the beam loss occurred gradually when the tune crossed the halfinteger downward, and rapidly in the other direction. These characteristics are qualitatively consistent with our experimental results.

According to Figs. 9(B) and 10(B), the rms beam size at each time agreed with the matched solution of the envelope equation with a fixed external field strength there. The growth of beam size before starting the beam loss was gradual and rapid in the downward and upward halfinteger crossings, respectively. Those characteristics of beam-size growth can be understood by the detuning effect depending on the beam size. If the beam size grows, the space-charge tune shift decreases and the depressed tune becomes higher. Therefore, the beam size gradually grows in the downward approach, keeping the depressed tune away from the half-integer value. On the other hand,
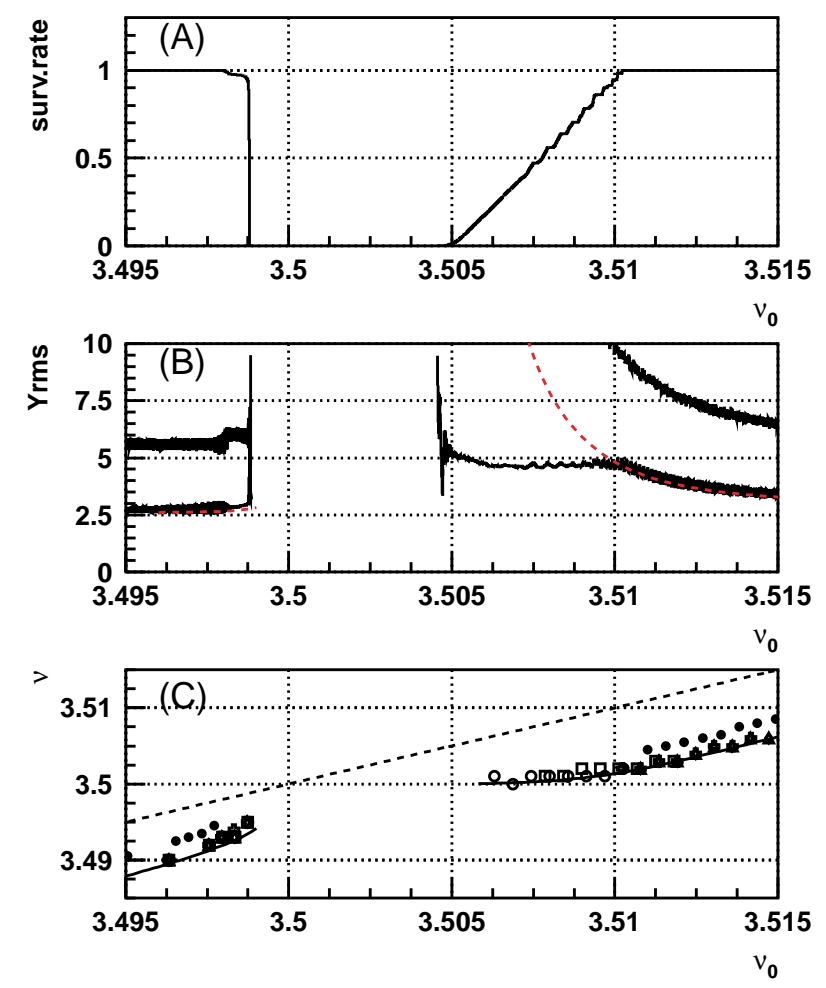

FIG. 9. (Color) Simulation results of resonance crossing, taking account of the space-charge effect. The distributions of macroparticles are uniform. (A) Ratio of the survival macroparticles. (B) The rms (lower) and maximum (upper) beam sizes. The dashed line shows the matched solution of envelope equation. (C) Observed tunes: the solid circles show the half of the quadrupole mode coherent tune and the white characters show the tunes of five single particles, whose initial amplitudes are 1-2 times the rms beam size. The dashed line is an unperturbed bare tune $\left(\nu_{0}\right)$. the beam size grows rapidly in the upward crossing because the beam-size growth accelerates the depressed tune approaching the half-integer value.

Figures $9(\mathrm{C})$ and $10(\mathrm{C})$ show the observed tunes. For a uniform beam, the tunes of single particles agreed with those calculated by Eq. (36), as expected. For a parabolic beam, those tunes were distributed around it. In the downward half-integer crossing, the decrease in tune was decelerated compared with the zero-current beam, and the depressed tunes of the test particles never cross the halfinteger. This is the detuning effect due to beam-size growth.

Figures 9(B) and 10(B) also show the maximum beam sizes, $y_{\max }$. They were calculated by

$$
y_{\max }=\tilde{Y}_{m} \frac{y_{99 \%}(0)}{y_{\mathrm{rms}}(0)},
$$

where $\tilde{Y}_{m}$ is the maximum value of the matched beam rms size with respect to $s ;\left[y_{99 \%}(0), y_{\mathrm{rms}}(0)\right]$ are the $99 \%$ and the rms beam sizes observed in a simulation at $s=0$. The maximum beam sizes are different between a uniform beam and a parabolic beam, which causes the difference in the beam-loss threshold between distributions.

It can be said that the threshold of the beam loss is independent of the aperture limit or COD in the upward half-integer crossing, because of the rapid characteristic of the beam-size growth. On the other hand, the threshold of beam loss depends directly on them in the downward half-integer crossing, where the beam size grows gradually.
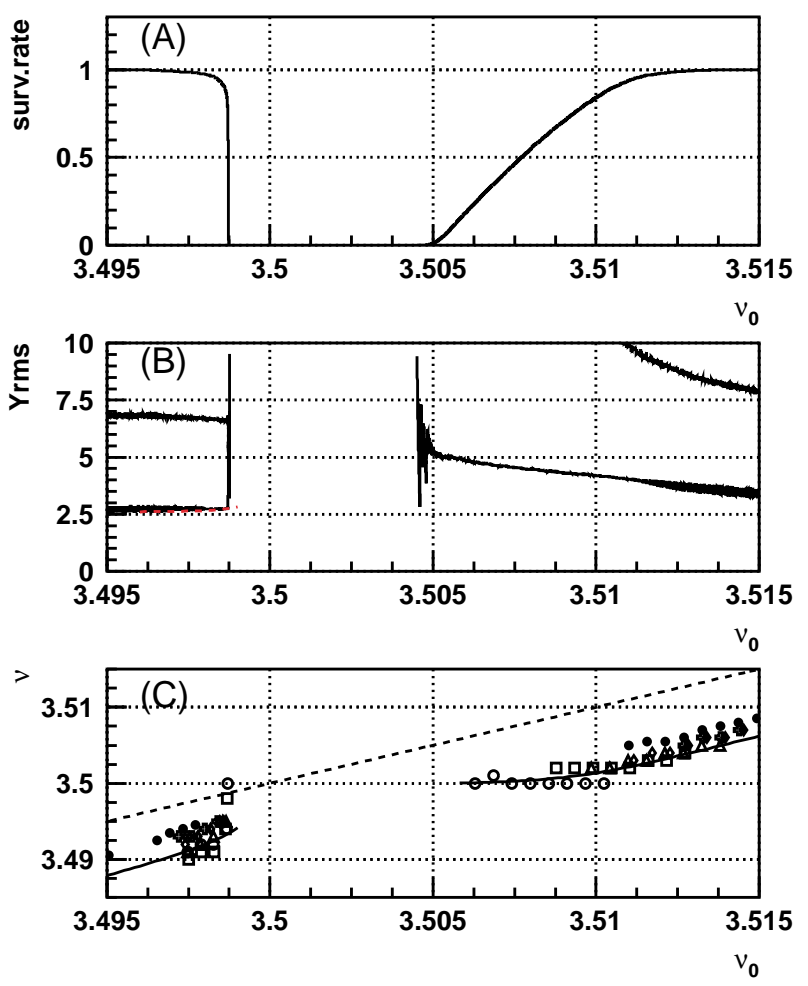

FIG. 10. (Color) Same as Fig. 9 but for a parabolic beam. 

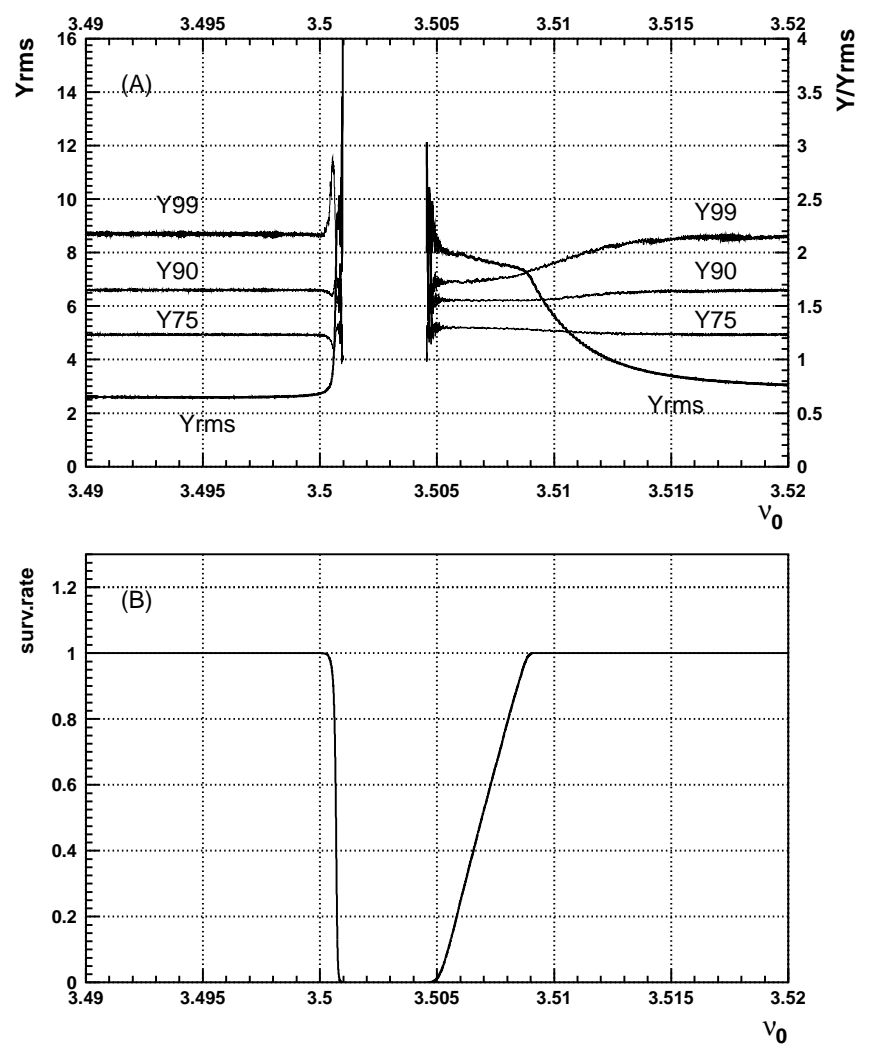

FIG. 11. (A) Ratio of $99 \%, 90 \%$, and $75 \%$ beam sizes relative to the rms size in a simulation with a large aperture limit. The initial distribution is parabolic. The absolute values of the rms sizes are also shown. (B) Fraction of the survival macroparticles.

In order to observe the change in the distribution with beam-size growth, we ran another simulation with a larger aperture limit (and a little larger intensity) starting with a parabolic distribution. Figure 11 shows the ratio of $99 \%$, $90 \%$, and $75 \%$ beam sizes to the rms beam size. In downward crossing, the ratio of $99 \%$ to the rms beam size began to decrease after the growth of the rms beam size exceeded $\sim 20 \%\left(\nu_{0} \sim 3.513\right)$. That means, those particles with a large amplitude contribute less to the growth of the rms beam size. On the other hand, that ratio became larger in the upward crossing.

\section{E. Summary}

We developed a multiparticle simulation on the approximation of the sheet beam and thin lens and reproduced the experiments of the half-integer tune crossing. The behaviors of the beam loss were gradual in the downward and rapid in the upward cross, as in the experimental results.

The beam loss as well as the growth of the beam size was gradual in the downward and rapid in the upward crossings. That behavior of the beam-size growth was the same as the results from Sacherer's analysis, which is based on an approximation of uniform external focusing. It can be explained by a decrease in the space-charge effect due to the beam-size growth itself. The rms beam size agreed with the matched solution of the envelope equation independent of the beam distribution, even if we took account of alternating-gradient focusing. Though the rms beam size is independent of the beam distribution, the threshold of the beam loss depended on it because the condition of beam loss relates to the full beam size.

The change in distribution with the beam size growth was observed in a simulation with a large aperture limit, starting with a parabolic distribution. In the case where the tune approached the half-integer downward, the charge density at the beam center became thinner. On the other hand, the full beam size grew rather than the rms beam size.

\section{DISCUSSION AND CONCLUSION}

As described in Sec. II, we measured the beam current in the HIMAC synchrotron when the vertical bare tune approached a half-integer by changing the strength of the defocusing quadrupole. With exciting the gradient error field as a source of the half-integer resonance, beam losses due to that resonance were observed there. In order to understand the experimental results, the beam losses in the experiments were reproduced in a one-dimensional multiparticle simulation with a thin-lens focusing model, as described in Sec. III.

As shown in Fig. 12, the results from both the experiments and simulations show that the beam loss occurred gradually when the tune approached a half-integer downward, and rapidly in the other direction. In addition, comparing Figs. 12(A) and 12(B), the bare tune where beam loss occurred in such processes was shifted higher when the initial beam intensity was high. In other words, the space-charge-induced tune shift changed the tune where a beam loss occurs due to the half-integer resonance in a synchrotron. This fact was verified experimentally in this work for the first time.

In the simulation, the beam size near a half-integer tune was observed at the center of a defocusing magnet every turn. The result showed that the growth of the beam size, in addition to the beam loss, occurred gradually when the tune crossed a half-integer downward, and rapidly when it crossed upward. Those characteristics agree with Sacherer's analysis. When the betatron tune decreases to meet a half-integer, a growth of the beam size occurs and the charge density becomes lower. Thus, the depressed tune stays away from a half-integer. This feedback stabilizes the beam above the zero-current stop band. After some particles of a beam reach the aperture of the vacuum chamber, beam loss starts, which lowers the charge density to keep the depressed tune away from the half-integer. This is why the beam loss and growth occurred gradually above the half-integer tune. On the other hand, when tune approaches a half-integer upward, both the beam-size growth and the beam loss make the depressed tune approach the 

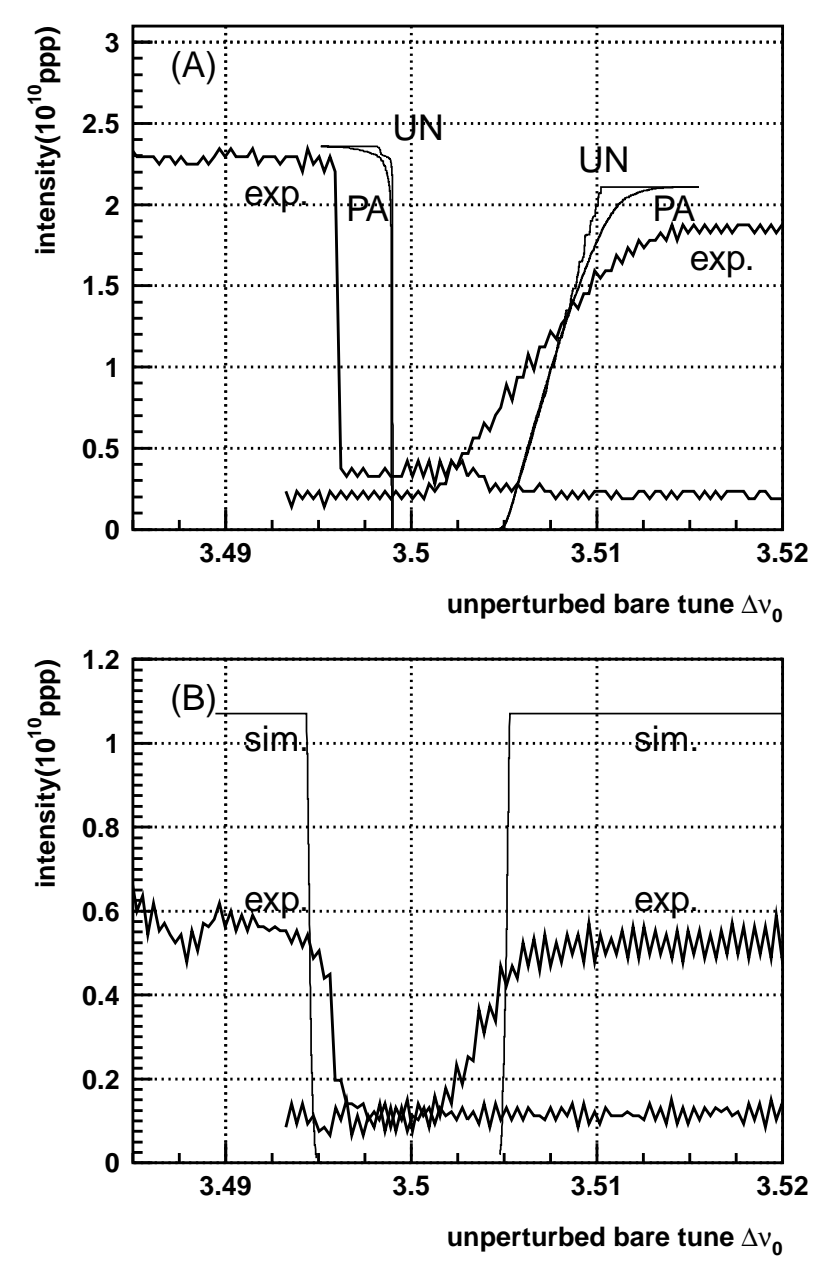

FIG. 12. Comparison of the beam loss waveform between the simulation (thin lines: UN for uniform beam and PA for parabolic beam) and experimental results (thick jagged lines). In (A), the vertical axis for the simulation data is scaled so as to have the same coherent quadrupole mode tune, using $\Delta \nu_{q} / 2=$ $0.0028 / 10^{10} \mathrm{ppp}$, while it is arbitrary in (B).

half-integer more, so that the beam is lost rapidly. From the characteristics of the beam-size growth, it can be said that the tune where the beam loss occurs directly depends on the aperture limit in the downward half-integer crossing, and is the same in the upward crossing whether the aperture limit is large or small.

We numerically solved the envelope equation for the rms beam size with any boundary condition in which the rms size and its derivative do not change after a revolution. That is called a matched solution. The rms size in the simulation, when it was less than the aperture limit, was in good agreement with the matched solution of the envelope equation, independent of the distribution (Figs. 9 and 10). In addition, the tunes of single particles in a uniform beam agreed with those calculated from the matched solution. Thus, the growth of the rms beam size due to the halfinteger resonance can be estimated by solving the rms envelope equation. It is independent of the distribution.
On the other hand, the beam loss is not determined by the rms beam size, but by the full size, so that the tune where the beam loss occurs depends on the distribution. Actually, the tune where the beam loss occurred differed between a uniform beam and a parabolic beam in the simulation. Therefore, we must know the distribution, or full beam size, to estimate the tune where beam loss occurs accurately. According to Fig. 11, the ratio of $99 \%$ to the rms beam sizes is constant unless the rms size grows more than $\sim 20 \%$ of the initial value (far from the half-integer tune) when the bare tune approaches the half-integer downward.

If the aperture limit exists near the full beam size, beam loss occurs when the beam size growth is still very small. In such a situation, the full beam size is expected to be proportional to the rms size until the beam loss begins. Therefore, if the distribution of injected beam is known, the condition of beam loss can be estimated from the initial value of the full size $(Y)$, the rms beam size $(\tilde{y})$, aperture limit $\left(Y_{\text {lim }}\right)$, and the maximum value of rms beam size $\left(\tilde{y}_{\max }\right)$ calculated by the envelope equation as a function of the bare tune and the beam intensity. That is, the beam loss occurs when

$$
Y_{\lim }<\frac{Y}{\tilde{y}} \tilde{y}_{\max }
$$

is satisfied.

If the aperture is sufficiently large, we must take into account the change in the distribution in order to estimate the tune where beam loss occurs. In a simulation of the downward half-integer tune approach, the charge density at the beam center decreases faster than that of the beam tail, so that the full beam size does not grow in proportion to the rms beam size. Thus, the tune where the beam loss occurs becomes closer to the half-integer tune than evaluated by Eq. (42). In the upward approach, the full beam size grows faster than does the rms size, where the beam loss occurs far from the half-integer.

The change in the distribution can be understood by the tune of individual particles, depending on their amplitudes, where "amplitude" means that of real space oscillation, not the phase-space area (emittance). In the downward approach to a half-integer tune, the tunes of the individual particles with small amplitude are closer to the half-integer, so that they grow faster than the others and the density at the beam center becomes low faster than the tail density. However, as the simulation showed in Fig. 11, this process does not necessarily cause beam loss because the tunes of those particles increase with their amplitude's growth. This is why the incoherent model of beam loss is not self-consistent. Figure 13 shows that particles with small amplitude contribute to the growth of the rms beam size more than large-amplitude particles. In Fig. 13, the Twiss parameters and emittances of four particles are calculated as functions of the bare tune by fittting the phase-space trajectory over 500 turns to $\gamma y^{2}+2 \alpha y y^{\prime}+\beta y^{\prime 2}=\epsilon$. The growth of beta functions is faster for those particles with 

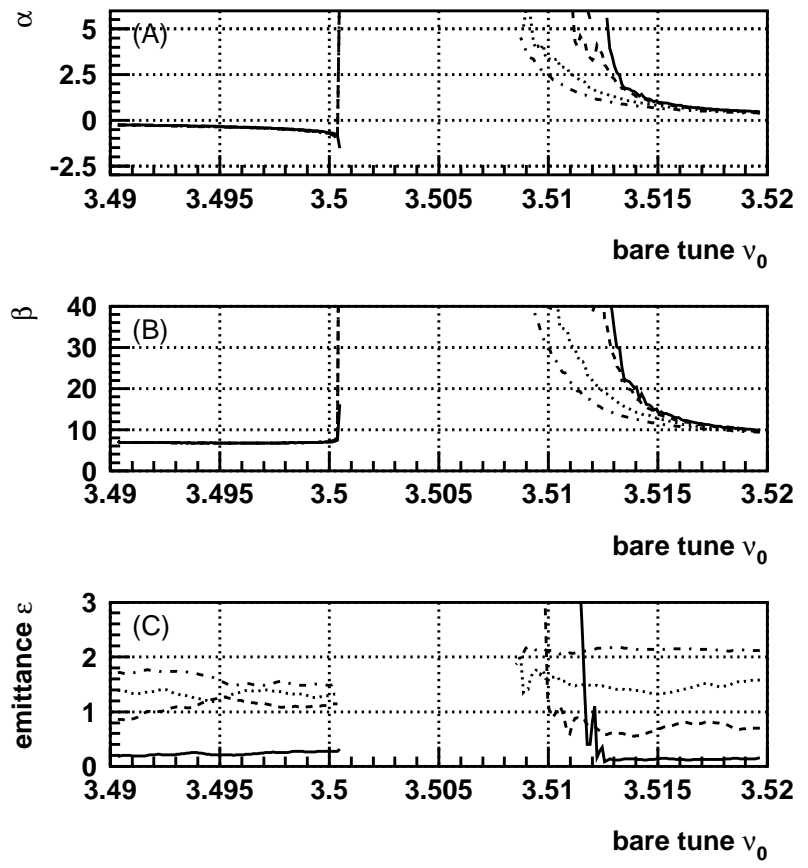

FIG. 13. Twiss parameters and emittances of four macroparticles, which were calculated by fitting the 500 turn phase trajectory to $\gamma y^{2}+2 \alpha y y^{\prime}+\beta y^{\prime 2}=\epsilon$. The solid line corresponds to a macroparticle with the lowest initial emittance.

a small emittance than for those with a large emittance. In the upward approach, the particles with large amplitude have tunes closer to the half-integer. Since the beam size growth occurs rapidly to cause a rapid beam loss, the change in the distribution is not important in this case.

In summary, we experimentally investigated the behavior of a beam near a half-integer tune with the help of a multiparticle simulation. The results showed that:

(i) The space-charge effect on the condition of halfinteger resonances was observed experimentally for the first time. In the presence of the space-charge effect, the bare tune where beam loss occurred due to the half-integer resonance became higher.

(ii) The beam size grows gradually when the tune approaches a half-integer value downward. Therefore, the tune where beam loss occurs due to the half-integer resonance depends directly on the aperture in such a process. On the other hand, the beam size grows rapidly in the upward approach.

(iii) An analysis of a half-integer resonance using the rms envelope equation gives a fairly good explanation to an actual beam in a synchrotron, where the external field employs an AG focusing scheme.

(iv) When the tune approaches a half-integer value downward, the beta function becomes larger, especially for particles with a small emittance, whose depressed tunes are nearer to the half-integer than the other particles. This is an incoherent picture. However, the growth of the beta function for those particles is limited because of the detuning effect.

\section{ACKNOWLEDGMENTS}

The authors would like to thank Dr. K. Noda and Dr. M. Kanazawa for their support in HIMAC experiments, and Dr. M. Ikegami and Dr. H. Okamoto for their helpful advice in the analysis of coherent motions. The authors thank Dr. D. Arakawa and Dr. T. Toyama who helped design the quadrupole beam monitor, and also K. Sato, S. Ninomiya, A. Mochihashi, and T. Oki who allowed us to use their rf-quadrupole kicker in our experiments. This work was carried out as a part of the Research Project with Heavy Ions at NIRS-HIMAC.

[1] E. D. Courant and H. S. Snyder, Ann. Phys. (Paris) 3, 1-48 (1958).

[2] M. Sands, SLAC Report No. SLAC-121 UC-28(ACC), 1970.

[3] F. J. Sacherer, Ph.D. thesis, University of California, Berkeley (UCRL-18454, 1968).

[4] R. Gluckstern, in Proceedings of the 1970 Proton Linear Accelerator Conference, Batavia, IL (Fermilab, Batavia, IL, 1970), p. 811.

[5] I. Hofmann, Phys. Rev. E 57, 4713 (1998).

[6] I. Hofmann et al., IEEE Trans. Nucl. Sci. 32, 2264 (1985).

[7] S. Machida, Nucl. Instrum. Methods Phys. Res., Sect. A 309, 43 (1991).

[8] D. Neuffer et al., in Proceedings of the Particle Accelerator Conference, San Francisco, 1991 (IEEE, Piscataway, NJ, 1991), p. 1893.

[9] R. Cappi et al., in Proceedings of the Particle Accelerator Conference, Washington, DC, 1993 (IEEE, Piscataway, NJ, 1993), p. 3570.

[10] R. Baartman, in Proceedings of the Workshop on Space Charge Physics in High Intensity Hadron Rings, 1998 (BNL, Upton, NY, 1998), p. 56.

[11] F. J. Sacherer, IEEE Trans. Nucl. Sci., 18, 1105 (1971).

[12] Y. Hirao et al., Report No. HIMAC Report No. NIRS-M89 HIMAC-001, 1992.

[13] M. Chanel, CERN Report No. CERN/PS 96-16(AR), 1996.

[14] N. Araki et al., in Proceedings of the 10th Symposium on Accelerator Science and Technology (JAERI, Hitachinaka, 1995), p. 272.

[15] T. Endo et al., in Proceedings of the Asian Particle Accelerator Conference, Tsukuba, Japan, 1998 (KEK, Tsukuba, 1998), p. 468; K. Sato et al., in, Proceedings of the European Particle Accelerator Conference, Stockholm, 1998 (Institute of Physics, Bristol, UK, 1998), p. 1330.

[16] L. J. Laslett and L. Resegotti, in Proceedings of 6th International Conference on High Energy Accelerators, Cambridge, MA, 1967 (MIT and Harvard University, Cambridge, MA, 1967), p. 150; B. Zotter, Nucl. Instrum. Methods 129, 377 (1975).

[17] W. Hardt, in Proceedings of 6th International Conference on High Energy Accelerators, Cambridge, MA, 1967 (Ref. [16]).

[18] W. H. Press et al., Numerical Recipes in FORTRAN (Cambridge University Press, Cambridge, 1992), pp. 704-707. 